\title{
Evolution of Mobility in Future Wireless Networks
}

\author{
Ajay Rajkumar \\ Alcatel-Lucent, 600 Mountain Avenue, Murray Hill, NJ, USA; \\ e-mail:ajay.rajkumar@alcatel-lucent.com
}

\begin{abstract}
With an ever increasing appetite for data over wireless networks, one is forced to think where the additional capacity in the wireless networks come from to carry the traffic? Some would argue that additional spectrum needs to be made available or a substantial increase in spectral efficiency has to come by. However, notwithstanding the above, an additional area that has important implications is to evolve seamless and non-seamless session mobility across disparate access technologies such as WiFi to offload some of the traffic to these alternate networks. Similarly, selective handover of specific flows across smaller cells such as Femto and Pico cells of the same access technology will have an impact on the overall capacity of the network. This not only will improve the capacity of these networks but may also enhance the quality of experience for users.
\end{abstract}

Keywords: 3GPP, LTE, data offload, WiFi, WLAN, femto, pico, small cells, smart phones, local breakout, multi access connectivity, IP flow mobility.

Journal of Cyber Security and Mobility, 101-115.

(c) 2012 River Publishers. All rights reserved. 


\section{Introduction}

In the last few years, wireless data networks have not only seen an evolution from an architectural standpoint but have also seen a significant transformation in the type of data services that are being provided on these networks. The transformation has been from circuit switch voice networks to complete packet data networks. Obviously, as these networks transition from circuit switched to packet data networks both these types of networks will co-exist where data intensive services will use the higher bandwidth networks and delay sensitive services will continue to use CS networks especially for services such as voice. Nevertheless, there have been many drivers for such a transformation starting with the antenna technology to the efficient transmission of data over these networks to the evolution of efficient architecture for these data networks. Most of all, the growth transformation has come from the advent of vast array of smart phones and the whole revolution in the applications aka "Apps" that sit on these smart phones.

These apps enable users to browse the web, to watch streaming video, to get real-time traffic updates on their mobiles, and have access to useful tools and data from the corporate intranets and the internet alike, which in turn spur the demand. The demand has also come because of the competition which has led to attractive prices for such services. However, with demand has come the need to satisfy.

According to a recent press announcement by AT\& ${ }^{1},{ }^{1}$ in the third quarter of 2011 alone there were more than three hundred million WiFi connections made by users, which amounts to more than 37 connections every second. This is nearly three times more connections on their network as compared to the same period in 2010. The above underscores that cellular macro networks are being supplemented by WiFi and other small cell networks for capacity needs. This may be due to the need for faster connections or fatter connections at the time when cellular network is not able to support the desired data rates or because the data consumed on these networks does not account for usage under various plans the user may be subscribed to. Regardless, of the reason this highlights the importance of data connections that are offloaded to other access technologies and results in the overall capacity increase in

${ }^{1}$ AT\&T Press Release, PRNewswire, October 24, 2011. 
the network. We would argue that in the future, this type of access would become even more critical as networks try to satisfy the need for increased bandwidth.

\section{Mobile Data Growth: The Driver}

As one models the projected load on these wireless networks, it quickly becomes apparent that bandwidth requirements will far outpace what the networks could currently support especially in the densely populated urban areas. On the face of it, Shannon's Law would contend that the available spectrum may not be sufficient to support the demand for bandwidth. These trends are exponential and will require some clever solutions. To alleviate this onslaught of bandwidth demand, besides increasing channel bandwidth mechanisms need to be devised that will increase the effective bandwidth available to consumers.

Some would argue that to alleviate this problem, the key is to increase available spectrum significantly for cellular services. With increase in channel bandwidth, obviously standards need to be defined with wider channels (20 MHz and higher) so that higher modulation schemes can be used. However, our belief is that this alone will not solve the problem. There are many different elements that are needed to increase the overall capacity in the network. Some of these mechanisms would surely be using Advanced Antenna configurations such as adaptive beam-forming, uplink and downlink Multiple-Input Multiple-Output (MIMO) transmission with or without feedback and Inter Cell Interference Coordination (ICIC) and enhanced ICIC. Another powerful technique to increase capacity is the deployment of Heterogeneous Networks (HetNets) using Pico and Femto cell in addition to macro cells in same geographic urban areas for indoor range extension in buildings, hole-filling of coverage within hot spots, or just supplement capacity.

In this paper, we will argue that yet another technique that may have even more significant impact on the available bandwidth within any geographic area is seamless and intelligent offload of data services. Offload could be either to a different access technologies such as WiFi or to the same access technology but from a macro network to a Pico or Femto network. It is important to note that the emphasis we are drawing is on both seamless and non-seamless intelligent mobility of services and flows. The proliferation of WiFi networks 
in homes and offices has made such techniques possible though challenges remain and we will explore some of them in the coming sections.

There is an array of devices that are being introduced into the market place. These range from feature phones that have limited capability for web browsing but are excellent phones for voice applications to smart phones such as blackberry which are good for email and data applications, to more advanced smart phones with integrated applications for web browsing to streaming video such as iPhone and Android based phones. Finally, tablets are a new breed of devices that sit in between laptops and smart phones in terms of form factor but in terms capabilities they are as capable of consuming large amount of bandwidth. From the data it is clear that depending upon the form factor of these devices, the ability to consume data for similar types of applications can vary significantly. This can range from $80 \mathrm{MB}$ per month on feature phones to almost 2 GB per month on tablet size devices.

We also notice that user trend for data growth is moving towards real-time mobile applications with streaming video, music, and real-time applications expected to gain even greater traction. Trends also indicate that data consumption on smart phones is increasing at a much faster rate than other types of handsets. There could be many factors for such trends. In our view, seamless integration of applications in these devices, a better graphical user interface, seamless connectivity to the network regardless of the types of access that may be available to the device are the key factors. These factors would become even more important as we aspire to have access to ubiquitous broadband connectivity irrespective of whether it is from any array of the different types of cellular network or WLAN hotspots or home networks.

\section{What Does Industry Mean by Traffic Offload and Specifically WLAN Offload?}

Traffic offload can be characterized by many different requirements. Some of these requirements are imperatives for the service providers and the others are requirements from a user perspective. At the outset it becomes clear that both seamless and non-seamless mobility solutions are important as each has relevance in different scenarios. These scenarios need to be built into the network architecture with minimum impact on network and no change to existing 
deployed equipment and yet not create inefficiencies in the network. This has a significant dependence on the end user devices of all types. Typically, for such schemes to work, one would see dual or tri-mode devices which can drain battery on these phones rapidly and render them as unattractive propositions. Minimizing constraints on devices could help faster adoption of such offloading solutions and help service providers accomplish the goal of increased capacity in the network. This also brings us to the role of intelligent offloading. Use of policies to intelligently and automatically choose the type of access network and then a specific network out of a list of networks is essential for the seamless offload to be effective.

One of the impediments for seamless mobility across disparate technologies and especially WLAN is authentication. Most WLAN networks may not use the same credentials that are used to authenticate a user on their cellular network. For true seamless operation, a user should be automatically authenticated based on credentials stored on one's Universal Integrated Circuit Card (UICC) using SIM/USIM authentication. Moreover, once access authentication has been granted, to further get services from the provider core network transmission over-the-air needs to be secured by strong encryption.

Types of services or family of services that are needed under WiFi are as follows:

- Plain Internet access without VAS (Value Added Services): usually free access

- MNO services such as IP or Content services: needs authenticated and secured access

- Possibility of Service Continuity and Service Consistency: same PS services and capabilities should be available under WiFi as they are available under macro wireless coverage such as 3GPP based networks

- For instance, similar charging info available - this does not mean that billing under WiFi will be the same as under $3 \mathrm{GPP}$ but that charging information should be available

- Evolution to offload with mobility (seamless handover, always on status from UEs) 
106 A. Rajkumar

\subsection{Main Characteristics of Offload Scenarios}

Let us now try to characterize different offload scenarios. There are two major families of selective offload that can be distinguished. They are Seamless and Non-seamless offload.

Following are the characteristics for each of them.

\section{Seamless Offload}

- IP address preserved which enables "Always on" connectivity and Session Continuity

- Selected traffic moved to WLAN and back to cellular access without service impact or interruption

- Suits offload of real-time or near real-time delay/jitter sensitive applications

- Requires standard mechanisms because of mobility and handover aspects, that is, interoperability is ensured

- Traffic via PGW/GGSN mobility anchor which is essential for seamless handover

- Maintain non-offloaded traffic on 3GPP access so that there is a possibility to bring offloaded traffic back to $3 \mathrm{GPP}$

\section{Non-seamless Offload}

- IP address not maintained which implies that it enables only nomadic and non-mobility usage

- Selected traffic moved to WLAN and back to mobile access with interruption

- Suits offload of non real-time, that is, non delay/jitter sensitive applications

- Access to secure MNO services should be possible over WLAN as well which is similar to access over $3 \mathrm{GPP}$

- 3GPP EPC or packet core bypassed for data flows and yet Security and Service Consistency is maintained using EPC infrastructure

- Maintain non-offloaded traffic on 3GPP access with a possibility to bring offloaded traffic back to $3 \mathrm{GPP}$

- Policy framework can enhance the access network discovery and selection function in the form of guidance to UE about available networks 


\section{Traffic Offload}

As traffic offload becomes a viable solution that complements the macro cellular network instead of being looked at as a network that could supplement it, there are many different solutions that are evolving. However, there is a significant difference between the type of solution that is possible with the Rel 8 and Rel9 standard and the type that could be accomplished with yet another evolution in these standards.

\subsection{Seamless Offload using 3GPP Rel8 and Rel9}

Solutions for seamless offload have been evolving. Within the 3GPP Rel8 and Rel9 standard, it is not possible to make selective mobile data offload to WLAN in contrast to 3GPP Rel10 and Rel11. With Rel8 and Rel9 all the traffic must be moved from one access to another, that is, there is no simultaneous traffic on both $3 \mathrm{GPP}$ and WLAN accesses except during HO. Figure 1 shows architecture for a high level solution for a UE that supports LTE and WiFi and the network that supports seamless offload using Rel8 and Re19 standard.

Consequently, with these releases the following two scenarios are possible:

Scenario 1: Considering that a $3 \mathrm{G}$ or $2 \mathrm{G}$ UE is available with WiFi support then all GPRS traffic can be offloaded to WLAN. This scenario exists today and can be implemented in the wireless networks but only for non-seamless

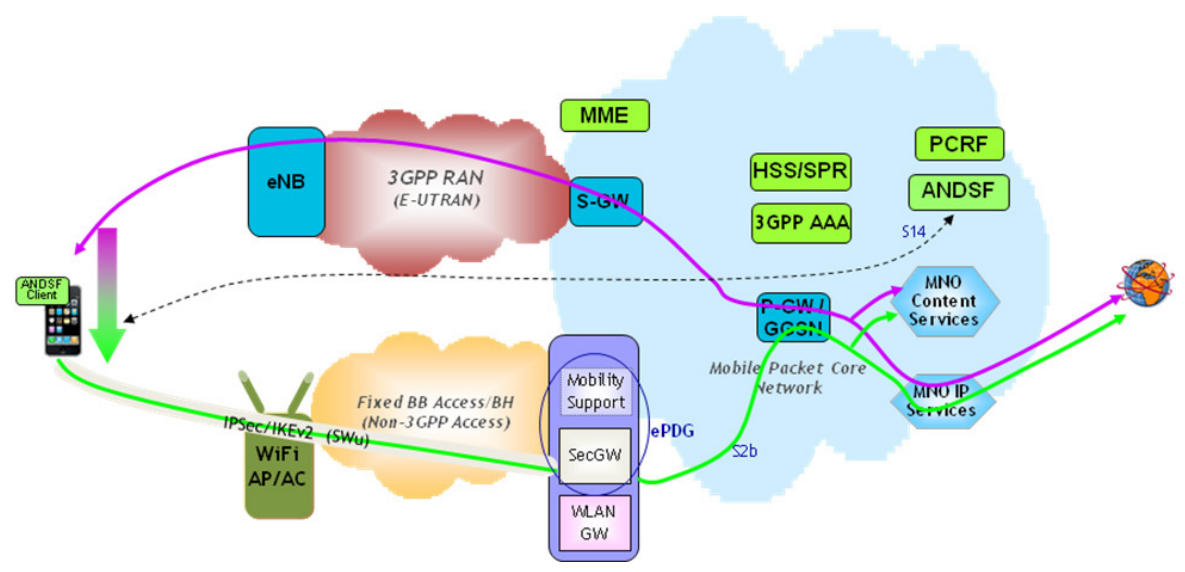

Figure 1 High level solution for LTE/WiFi UE seamless offload using Rel8/9. 


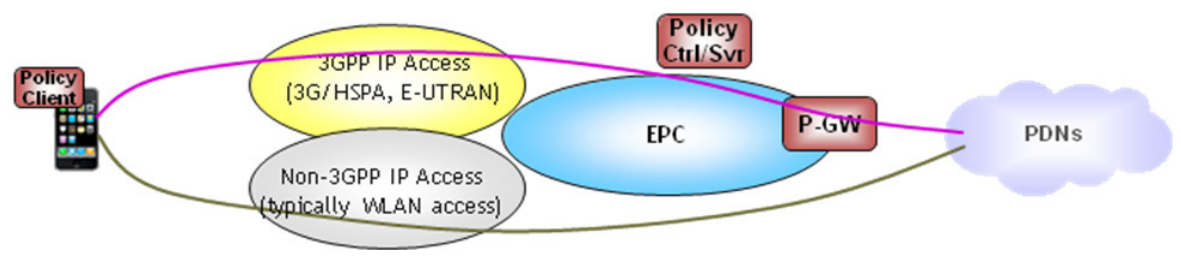

Figure 2 Non-seamless offload with policy support.

offload and without policy support. Usage of network based policy to guide the behaviour of a UE is a significant difference.

Scenario 2: Considering a UE with 3G, 2G, and LTE access technologies including WiFi then all Enhanced Packet System (EPS) traffic is moved to WLAN.

In both the above scenarios, the ability to revert back to the 3GPP network is a possibility in case the same anchor is being used. Note that for the above scenarios where traffic offload is between LTE and WLAN, deployments require an EPC with PGW as mobility anchor node for any seamless handover across these disparate technologies. This would mandate the use of ePDG and S2b interface to a PGW. Currently, two different mechanisms are supported for mobility between the access gateway and the anchor gateway. PMIPv6 based mobility is supported across technologies for Rel8/9 whereas, GTP based S2b option is supported in addition to PMIPv6 for Rel10.

\subsection{Policy Considerations for Seamless and Non-seamless Offload}

Policies are essential for an intelligent handover from one access technology to another. These policies would dictate under what conditions the offload to an alternative technology should take place. Intelligent handovers are defined where complete sessions do not move but individual flows could move to the most appropriate technology. This requires that the network first have the information regarding individual flows and secondly, have the ability to extract these individual flows and assign them to separate Packet Data Networks (PDN) with their own IP address and Quality of Service (QoS) metrics. An example of such selective offload would be that if a streaming session is being played and WiFi is available then just that streaming session be moved instead of the whole session. Similarly, if a delay sensitive application such as 
voice call is being made then the voice session remains on the cellular network. Another example would be where a large download is offloaded depending on the whether the user is roaming or on the home network. The above examples are specific to a single user whereas if the network is congested then policies could dictate the behaviour of the network across users as well.

The above is possible with the help of the policy infrastructure in terms of a Policy Charging and Rules Function (PCRF) that dynamically interacts with the PGW and the enforcement point in the network. Policies are required to give guidance to UE based on operator policies, user preferences, user subscription, and access conditions. These considerations can be handled statically and/or dynamically.

IEEE 802.21 Media Independent Handover Services [4] and Access Network Discovery and Selection Function (ANDSF) defined in 3GPP [2] are two different methods that have been defined in the standards that offer dynamic operation and knowledge of alternate access types available to a UE. IEEE 802.21 defines three services namely, Information Service, which is the closest to the ANDSF but also defines Event Services and Command Services. Event Services could provide the local operating environment information for different connections that a UE may have access to at any time.

Under the term "Policy" used for Network Discovery and Selection (NDS) several aspects are included though except ANDSF not all have been specified at this time. ANDSF defines Inter-system mobility policies, access network discovery information, and inter-system routing policies but the following have not been defined.

- Static Configuration: Operator provisioned and/or hard coded parameters in the Dual Mode (3GPP/WiFi) terminal

- Local Operating Environment (LOE): Radio environment information, quality of IP connection, application specific requirements, power considerations

- User Preferences: The end user must have some liberty to configure his/her terminal for WLAN interworking and offload

\section{Emerging Solutions}

Typically, offload solutions can offload traffic from both the Access Network and core Network but sometimes it may be useful to just offload the access 
network or the core network. For value added services anchoring the services to the core network is essential and for that reason only an alternate radio access network may be desired rather than the entire session moving to an alternate network. Similarly, it may be desirable to have the complete user session moved or only some specific flows be moved. We will discuss some of the scenarios that are currently being worked. These scenarios will be able to facilitate intelligent offload of sessions and flows that will enhance user experience and at the same time help service providers manage elevated bandwidth demand dynamically.

For local breakout of traffic, the schemes that are being developed are:

- Local IP Access (LIPA) [3]

- Selected IP Traffic Offload (SIPTO) [3]

Similarly, for simultaneous multiple access connectivity the following schemes are being developed:

- Multi Access PDN Connectivity (MAPCON) over S2x as shown in Figure 3. In this scenario, the initial PDN Connectivity (e.g., Internet session) with all its flows is moved seamlessly from 3GPP access to non-3GPP access while keeping Pink PDN Connectivity (e.g., Real-time session) on 3GPP access.

- IP Flow Mobility (IFOM) [1] can be divided into Host Based Mobility that uses S2c interface using dual stack and Network Based Mobility over S2a and/or S2b interfaces. This is shown in Figure 4. In this scenario, Brown IP Flow (e.g., video streaming) of a certain PDN Connectivity moved seamlessly from 3GPP access to same PDN Connectivity of non-3GPP access (already using a Red IP Flow (e.g., FTP Connectivity)) while keeping Purple IP Flow (e.g., Real-time session) also of same PDN Connectivity on 3GPP access.

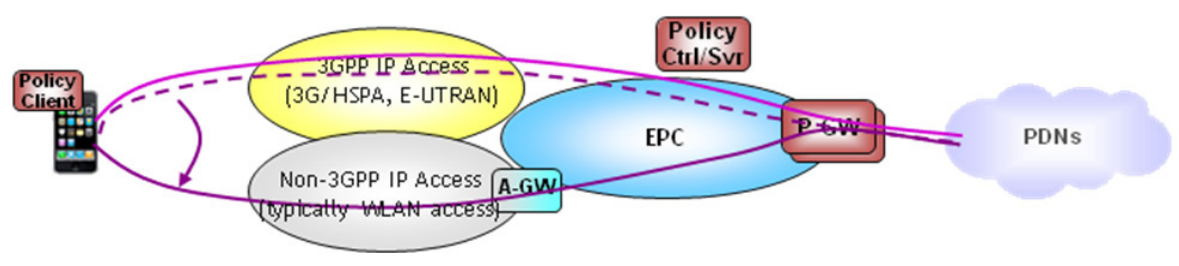

Figure 3 MAPCONN seamless offload, 3GPP Rel 10. 


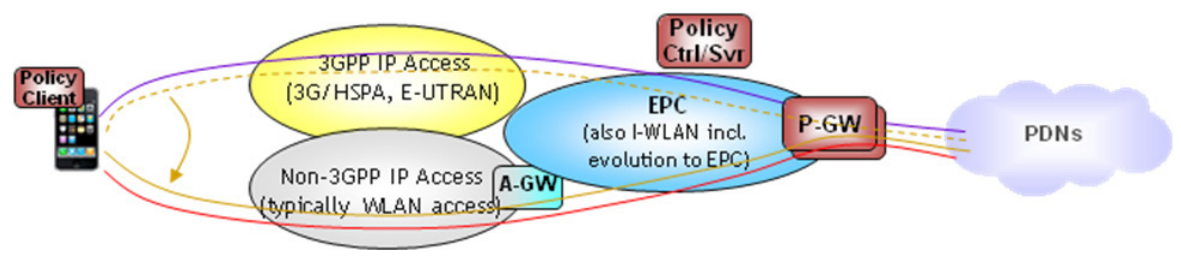

Figure 4 IFOM seamless offload.

\subsection{Host Based Mobility vs. Network Based Mobility}

Most of the networks have defined two types of mobility. One of them is called Host Based Mobility (HBM), which is defined when mobility is managed by a UE. In this form of mobility, UE has the mobility client which is responsible for initiating the handover from LTE to other access technologies such as CDMA based EVDO or WiFi as well as within the same access technology across the access gateway such as Serving Gateway (SGW) in LTE. This is in contrast to the other type of mobility called Network Based Mobility (NBM) where the mobility client is located in the network. The client is typically located on the access gateway in the network. This client on behalf of the $\mathrm{UE}$ is responsible of communicating and coordinating with the anchor point to make sure that the seamless handover across technologies or within the same technology takes place smoothly. Currently, IP Flow Mobility (IFOM) HBM is the standard solution. However, an NBM solution has some significant benefits with respect to HBM.

Some benefits to consider for Network Based Mobility solution are:

- NBM avoids use of Mobile IP (MIP) in the terminal which is considered to be a more dynamic ecosystem to manage. For instance, WiMAX based network defined both Client MIP typically used for host based mobility and Proxy MIP typically used with network based mobility. However, today all WiMAX network deployments use PMIP to support mobility. The reason being that CMIP terminals are not widely available and operators prefer that mobility control remains in the network.

- NBM avoids, in contrast to DSMIPv6 used by the S2c interface, the use of an IPSec tunnel between UE and PGW which is on a per user basis which could cause a scalability issue in particular for the PGW 
- NBM with PMIPv6 functionally just requires a single tunnel between the access gateway and the PGW. However, practically more than one tunnel may be used to accommodate all users connected to the network depending on the capacity of the PGW and redundancy mechanism and deployment strategy for a given network. For un-trusted access, an additional IPSec tunnel per UE is required between the UE and the ePDG. This means that a "tunnel in tunnel" solution must be supported by a UE that is based on DSMIPv6 over S2c interface which can be inefficient as compared to an NBM solution.

The NBM solution is seen as more appropriate for the PGW and EPC in general.

\subsection{Seamless Offload to Small Cells of Same Access Technology}

The issues for offload of traffic to Pico and Femto Cell are similar to WLAN Offload. However, some differences that can affect the architecture or the overall solution depend on answers to the following questions:

- Is inter-cell handoff between Pico/Femto and macro network supported?

- Does the traffic go through the same packet data network?

- Is the same authentication infrastructure used for both the macro network and the Pico/Femto cell network?

The above questions underscore that Pico and Femto Networks can either be viewed as an extension of macro cellular network from a mobility standpoint in which case all the above issues would be mute. On the other hand, these small cell networks could be considered as similar to WiFi networks except they have use the same access technology as the macro network and then all the scenarios for WiFi offload and the issues thereof come into consideration. The challenge for same technology small cell deployment scenario would be that the same radio as opposed to having dual-radio in the UE will need to communicate to two different networks. This will require some co-ordination even if the traffic does not go through the same PDN or the same authentication infrastructure is not used by the two networks. 


\title{
6 Conclusion
}

In the past decade, packet data networks have significantly evolved in many different aspects starting from bandwidth they support, to services they carry, to devices that connect to them. The continuation of this evolution requires that networks be able to communicate to a range of disparate networks both seamlessly and non-seamlessly. As we see it, multiple disparate access technologies available on the same UE in the same geography are already becoming a reality, so as a final thought, the question comes down to - can we overcome constraints and are such wireless networks practical such that individual applications flow over specific optimized disparate access networks simultaneously and adapt service flows seamlessly and dynamically to the available networks? We believe that technically such solutions can be developed though from a deployment standpoint it will take a number of evolutionary iterations in devices and wireless networks alike before we reach that end goal.

\section{Acknowledgements}

I will like to thank my colleagues at Alcatel-Lucent for discussions around this topic and other general aspects of evolution in wireless networks.

\section{References}

[1] 3GPP TS23.261: IP flow mobility and seamless Wireless Local Area Network data offload.

[2] 3GPP TS23.402: Architecture Enhancements for non-3GPP Accesses.

[3] 3GPP TR23.829: Local IP Access and Selected IP Traffic Offload (LIPA-SIPTO).

[4] IEEE Std 802.21-2008, IEEE Standard for Local and Metropolitan Area Networks Part 21: Media Independent Handover Services, IEEE, January 2009.

\section{Abbreviations}

\author{
AGW Access Gateway \\ ANDSF Access Network Discovery and Selection Function \\ APN Access Point Name \\ CMIP Client Mobile IP \\ DSMIPv6 Dual Stack Mobile IPv6 \\ ePDG enhanced Packet Data \\ GTP Generic Tunnelling Protocol
}


114 A. Rajkumar

HBM Host Based Mobility

HetNet Heterogeneous Networks

ICIC Inter-Cell Interference Co-ordination

IFOM IP Flow Mobility

LIPA Local IP Access

LOE Local Operating Environment

MAPCON Multi-Access PDN Connectivity

MIMO Multiple Input Multiple Output

MIP Mobile IP

MNO Mobile Network Operator

NBM Network Based Mobility

NDS Network Discovery and Selection Gateway

PCRF Policy Charging Rules Function

PDN Packet Data Network

PGW PDN Gateway

PMIP Proxy Mobile IP

QoS Quality of Service

SGW Serving Gateway

S2a Interface between PGW and Trusted Non-3GPP IP Access Network

S2b Interface between PGW and ePDG in the Un-trusted Non-3GPP IP Access Network

S2c Interface between PGW and the UE through the Un-trusted Non-3GPP IP Access Network

SIM Subscriber Identity Module

SIPTO Selected IP Traffic Offload

UE User Equipment

UICC Universal Integrated Circuit Card

USIM Universal Subscriber Identity Module

VAS Value Added Service

WLAN Wireless LAN 


\section{Authors' Biography}

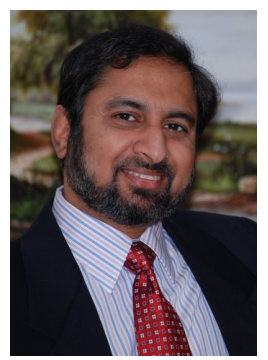

Ajay Rajkumar is Director, Americas Wireless Chief Technology Office at Alcatel-Lucent. He holds a BS (Honors) in Mathematics from Delhi University, MS in Computer Science from Pune University and received a Ph.D. in Computer Science from Courant Institute of Mathematical Sciences, New York University.

During more than nineteen year in the wireless industry, first at Bell Labs Research and then in Wireless Division research, Dr. Rajkumar has led and worked towards extending the cutting-edge of technology in modeling, service applications, network performance, end-to-end wireless network architectures, and various standards. He has worked on the evolution of flatter and efficient Network Architecture and Mobility Management for the Next Generation Networks such as LTE/SAE, UMB, and WiMAX. Dr. Rajkumar led the RAN architecture design for efficient service and application enablement, design of Mobile Edge-Content Delivery Network, EVDO Base Station Router design, and the first prototype for Push-to-Talk on CDMA Packet Data Networks. He was an early proponent and developer of IP Backhaul. He has more than two dozen patents received and pending.

Dr. Rajkumar is a Member of Alcatel-Lucent Technical Academy, Distinguished Member of Technical Staff, and a recipient of Bell Labs President's Award in 2002 and 2005. He is currently the Chair of the Eastern US Chapter of Alcatel-Lucent Technical Academy. In the past, he has chaired several international conferences. He has been an invited speaker at universities and industry forum.

Dr. Rajkumar also led the wireless industry to develop standards for maintaining seamless session across heterogeneous access networks especially for real-time applications thus enabling networks towards providing ubiquitous coverage. He was the founding Chair of IEEE 802.21 Working Group on Media Independent Handover Services and a member of IEEE 802 Executive Committee from 2004-06. 
